\title{
Improvement in two-dimensional barcode
}

\author{
SONAM WASULE ${ }^{1, *}$ and SHILPA METKAR ${ }^{2}$ \\ ${ }^{1}$ College of Engineering, Pune 411005, India \\ ${ }^{2}$ Electronics and Communication Engineering, College of Engineering, Pune 411005, India \\ e-mail: sonam191091@gmail.com; metkars.extc@coep.ac.in
}

MS received 24 March 2016; revised 2 September 2016; accepted 20 September 2016

\begin{abstract}
Barcode is one of the existing systems which is very fast in scanning and more accurate when compared with other coding systems. It is extensively used because speed of scanning the barcode is very high as compared with manual data entry. To increase the capacity of 2D monochrome QR code to 3 fold, 2D colour QR code is developed. The challenge in the development of colour barcode is in its decoding, since the intensity and depth of colours vary during the printing and scanning process. We need to understand the decoding process and make it insensitive to such variations. A lot of work has been already done to deal with such variations but acceptable results have not yet been achieved. The objective behind colour barcode is to increase the capacity to 3 fold as compared with 2D monochrome barcode. In this paper we proposed a novel approach that will increase the capacity of barcode beyond 3 fold and deals with decoding problem of intensity variation. In the proposed technique, quantization of grey levels is specified to handle the problem of intensity variation.
\end{abstract}

Keywords. Barcode; vector quantization; bit plane mapping; printing and scanning.

\section{Introduction}

Barcode is a representation of digital data that are encoded in it and can be read using an optical machine. It has several advantages over human performance like accuracy, speed and so on; hence barcode technology is used extensively. Barcodes are broadly used in automatic identification for a wide range of real time applications from its development in early 1970s [1]. In 2D barcodes, the information that is encoded will be stored in vertical direction as well as in horizontal direction. Recent developments in the barcode are pdf417 [1, 2], quick response, data matrix [3] and aztec. QR code is widely used barcode, worldwide. The advantages of 2D barcodes include less area, high embedding capacity, higher density and higher error detection and correction level [4]. 2D QR colour barcodes are primarily developed to increase the embedding capacity of the barcode to 3 fold over that using a single colour (black and white) barcode. The 3 -fold increase in the data rate is achieved by encoding independent data using cyan, yellow and majenta (CMY) channels. This data is printed by colour printer which uses CMY colourant channels while capturing devices uses red, green and blue sensing channels. This causes problem of intensity and depth variation during printing and scanning. The problem of colour interference

*For correspondence in 2D colour barcode is overcome by a Expectation Maximization Algorithm and Pilot Block Approach [5].

2D barcodes were originally used in Japan to track vehicle parts during the manufacturing process but now they are more popular in other areas such as marketing. 2D codes can connect users to many different types of data such as web pages, images, contact information, SMS text messages and more. 2D barcodes can be read by simply scanning them with a $\mathrm{QR}$ code reader app downloaded on a smartphone. 2D barcodes have been very useful in a number of industries including retail, products like books and other items. The development of colour barcodes is still challenging since the intensity and depth of colours vary during printing and scanning processes. We need to find a way to deal with intensity and depth variation in printing and scanning. In this paper, we have proposed a new method of encoding information using grey levels in order to increase capacity of $2 \mathrm{D}$ barcode as compared to $2 \mathrm{D}$ colour QR code and also with acceptable variation of intensity during printing and scanning.

\section{Recent explorations}

\subsection{Quick response $(Q R)$ colour barcode}

QR colour barcode uses three colours: cyan(C), magenta (M) and yellow (Y). QR colour code increases the data capacity up to three times as compared to QR monochrome 
barcode. In CMY model, also known as subtractive primaries, the white colour disappears on subtracting the three colours such as cyan, magenta and yellow. However, these three colours form a new colour called the Key colour that is black. These colours can vary in intensity to form 10, 12 or more colour sets as shown in figure 1 [6]. As the intensity of the colour varies, the colour barcode becomes more complex thus increasing the complexity of decoding algorithm. As three single colour barcodes are combined to form a single multi-colour barcode, interference between the colours of single-colour barcode takes place as shown in figure 1 [7].

\subsection{High Capacity Colour Barcode (HCCB)}

HCCB [8] uses eight colours as shown in figure 2. One of the latest developments in the barcode technology of colour barcode is Microsoft's High Capacity Colour Barcode (HCCB). With four or eight colours, colour barcodes are now used extensively in certain areas such as airports, print advertising and mobile phones [6].

HCCB has many advantages, such as high data capacity and data security and compression, over the traditional

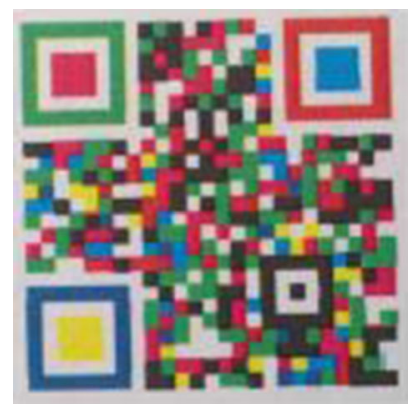

Figure 1. Colour QR code.

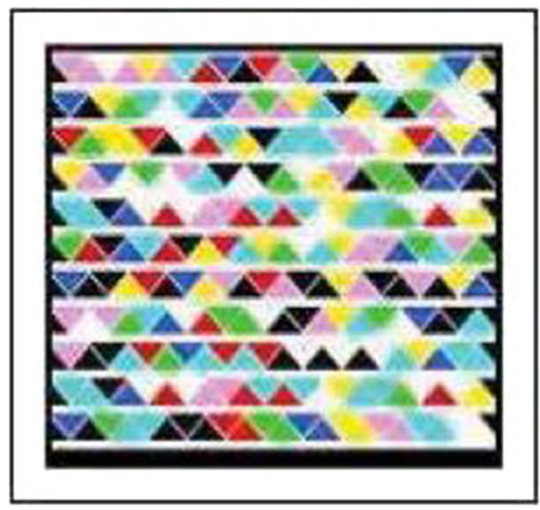

8 colour barcode storing 84 RAW bytes black and white barcodes. The development of colour barcodes is still challenging since the intensity and depth of colours vary during printing and scanning processes. Using eight colours, it became easier to develop the barcode further, increasing the capacity upto $70 \%$.

\subsection{Disadvantages of colour barcode}

The colour barcodes provide increased data rates compared with 2D monochrome barcodes by encoding data in the colour of small shapes (triangles/rectangles). However, the use of colours for encoding poses challenges in the presence of inevitable tonal variations in the print and capture processes which cause changes in the colours and to make decoding feasible. A separate algorithm needs to be implemented in order to decode the barcode, due to the interference among the colours [7]. Due to the interference, the decoding algorithm becomes more complex [4-6].

\section{Theoretical background of proposed method}

In this section, the proposed method of encoding is explained. The proposed method uses grey levels in each module to represent the combination of binary bits. We created a barcode image inspired by the concept of bit plane mapping. Each module is represented by a chosen grey level, where a combination of ' $n$ ' bits is represented by a range of grey levels. Multiple sequences can be represented using bit plane mapping, such that, bit plane ' $n-1$ ' encodes first sequence, bit plane ' $n-2$ ' encodes second sequence and so on, bit plane ' 0 ' encodes the last sequence. We imagine a bit plane ' $n-1$ ' that contains modules encoding the binary bits of the first sequence. Behind this plane there lies bit plane ' $n-2$ ', and so on. This creates imaginary bit planes that are situated one

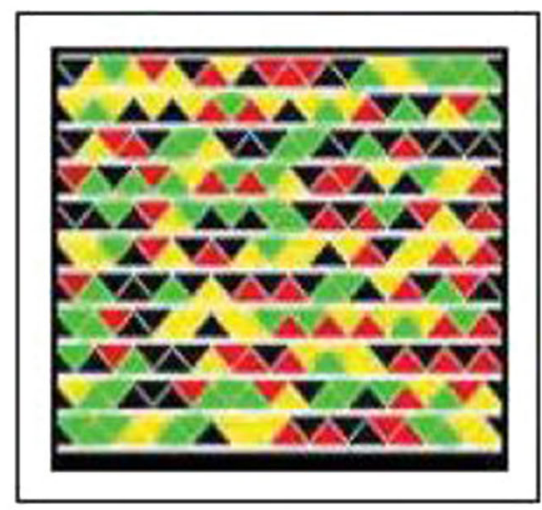

\section{4 colour barcode storing 58 RAW bytes}

Figure 2. The Microsoft tag barcodes. 
behind another. Here, we have developed a sort of representation in which grey levels are chosen for representing a combination of binary bits. Different grey levels are assigned to different combination of bits. Use of quantization in decoding increases the acceptance of intensity variation during scanning and printing. The proposed method uses grey level $0-255$, that is, we can encode eight sequences simultaneously in a single barcode but without any acceptance of intensity variation. If we reduce the number of sequences to be encoded then the acceptance level of intensity variation increases. QR code and Aztec code are extensively used because of easy decoding using mobile phones. The proposed technique adopted the standard QR barcode encoding method.

\section{Proposed data encoding technique}

A literature survey on 2D colour barcode brought about a new development to the barcode technology. The aim was to maximize the data density while reducing error. Using four and eight colours a new colour barcode was developed that could store more data than via $\mathrm{QR}$ code. The developed 2D colour barcode has many advantages, such as high data capacity, data security and compression, over the traditional black and white barcodes. The development of colour barcodes is still challenging since the intensity and depth of colours vary during printing and scanning processes.

We proposed a method to overcome shortcoming of colour barcode. Proposed method encodes multiple data

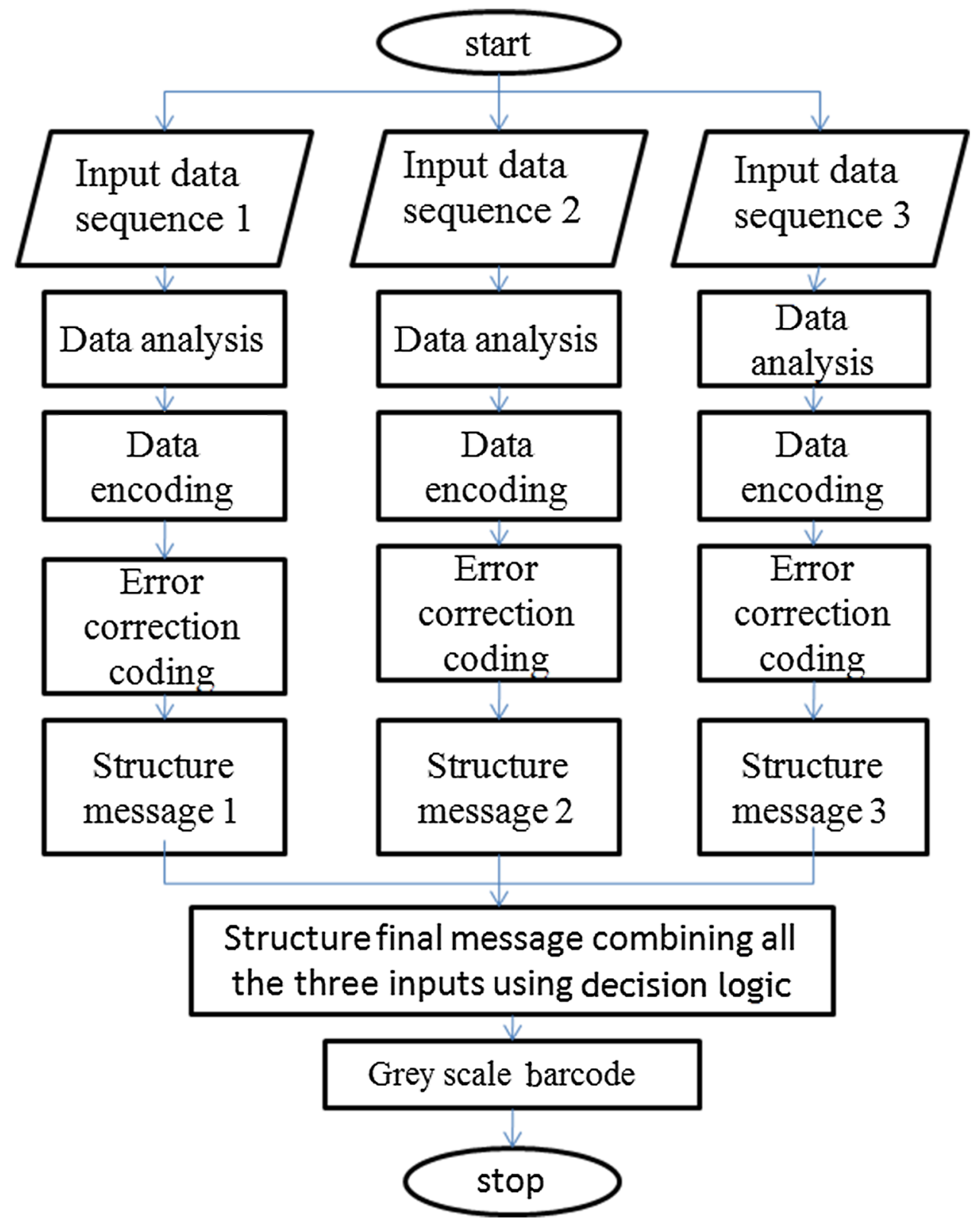

Figure 3. Proposed flow diagram to generate grey scale QR code. 
sequence in a single barcode, which increases the capacity of the barcode compared with monochrome barcode. We proposed a framework for extending monochrome barcodes to grey level barcode as shown in figure 3 . We can use this method to encode $\mathrm{n}$ independent data $m_{1}, m_{2}, m_{3}, \ldots m_{n}$, $(n \leq 8)$ contained in bit plane ' $n-1$ ', bit plane ' $n-2$ ', ...bit plane ' 0 ', respectively. Data are encoded in each bit plane, utilizing the error correction coding available to monochrome counterpart to allow error recovery for data extracted from the barcode. Note that the proposed framework is applicable to any monochrome barcode; the encoding rate of the monochrome counterpart can be increased utilizing imaginary bit planes using grey levels. The independent input data are converted into a binary sequence of bits by the conversion used in QR encoding, with the error correction bits. The final stream of bits obtained for each sequence $m_{1}, m_{2} \ldots m_{n}$ is used in combination (group) to encode it into grey level, taking corresponding bit from each sequence. The primary contribution of this paper lies in developing a new method that solves the problems faced by the colour barcode during decoding. This paper explains the proposed method for encoding three independent data, which can be further extended to ' $n$ ' independent data.

A QR code is a special type of barcode that can encode information like numbers, letters and Kanji characters. Figure 3 indicates different steps involved to generate grey scale QR code and these are explained in following sections.

\subsection{Data analysis}

A QR code encodes a string of text. The QR standard has four modes for encoding text: numeric, alphanumeric, byte, and Kanji. Each mode encodes the text as a string of bits (1s and $0 \mathrm{~s}$ ), but each mode uses a different method for converting the text into bits, and each encoding method is optimized to encode the data with the shortest possible string of bits. The numeric mode is for the decimal digits 0-9. Alphanumeric mode is for the decimal digits 0-9, uppercase letters (not lowercase!) and the symbols $\$, \%$, *, ,,.,$+- /$, as well as a space. The byte mode, by default, is for characters from the ISO-8859-1 character set. The Kanji mode compresses Kanji characters more efficiently. It is also possible to use multiple modes within the same QR code.

\subsection{Data encoding}

After selecting the appropriate encoding mode for the text, the next step is to encode the text. The result of this step is a string of bits that is split up into data codeword which are each 8 bits long. Each encoding mode is designed to create the shortest possible string of bits for the characters that are used in that mode. Each mode uses a different method for converting the input text into a string of bits.

\subsection{Error correction coding}

Error correction codeword allows QR code readers to detect and correct errors in QR codes. After creating the string of data bits that represent the text, these bits are used to generate error correction codeword using a process called Reed-Solomon error correction.

\subsection{Structuring Independent Messages (structure message 1, structure message 2...structure message n)}

After the Reed-Solomon error correction, the data blocks and error correction codeword are interleaved according to the QR code specification. Firstly, the data blocks are interleaved and then the error correction codeword.

\subsection{Structuring final message}

Final stream of bits is generated using the decision logic explained in section 5 .

\subsection{Grey scale barcode (grey scale $Q R$ code)}

The final stream of bits is then placed in the QR code matrix along with the required function patterns. A function pattern is a non-data element of the $\mathrm{QR}$ code that is required by the $\mathrm{QR}$ code specification, such as the three finder patterns in the corners of the QR code matrix.

\section{Decision Logic to combine independent data sequence}

To combine independent message sequences into a single structure, decision logic is proposed which is based on vector quantization. This section explains quantization of grey levels to map independent sequences into a single structure. This section has also proposed encoding and decoding decision logic to combine independent monochrome barcodes into a single grey scale QR code and to retrieve independent monochrome barcodes from a single grey scale barcode respectively.

\subsection{Vector quantization}

Vector quantization is performed over block of grey levels. The encoding logic for combining multiple barcodes into a grey scale barcode determines best representing grey level 
for a particular group of grey levels. The decoding logic takes the same representation of grey levels instead of its own, in order to obtain multiple monochrome barcodes encoded in a single grey scale barcode.

\subsection{Data encoding decision logic}

If $n$ sequences are to be encoded then number of combinations that can be formed is $2^{n}$, that is, eight combinations for three sequences. Each combination of bit is assigned a grey level intensity, such that 256 grey levels are assigned with constant gap starting from 0 to 255 as shown in figure 4. This type of assignment is done at the time of encoding, which will be helpful at the time of decoding by introducing quantization in decoding. Applying quantization in encoding makes decoding tolerable to intensity variations that occur at the time of printing and scanning.

The grey level values assigned to the binary bit combination are not standard values. Figure 4 specifies the assignment of grey levels to various binary bit combinations for encoding of three independent data.

The following equations are used to assign grey levels to the combination of bits.

$$
\begin{aligned}
& c \_d=\operatorname{int}\left(256 /\left(2^{n}-1\right)\right) \\
& g \_l=c \_d \times \text { decimal equivalent of binary combination } \\
& \quad(\text { refer figure } 4)
\end{aligned}
$$

where, $c \_d=$ common difference for grey level representation

$\mathrm{n}=$ number of independent data sequences to be encoded int $=$ integer value after division

$g \_l=$ grey level assigned

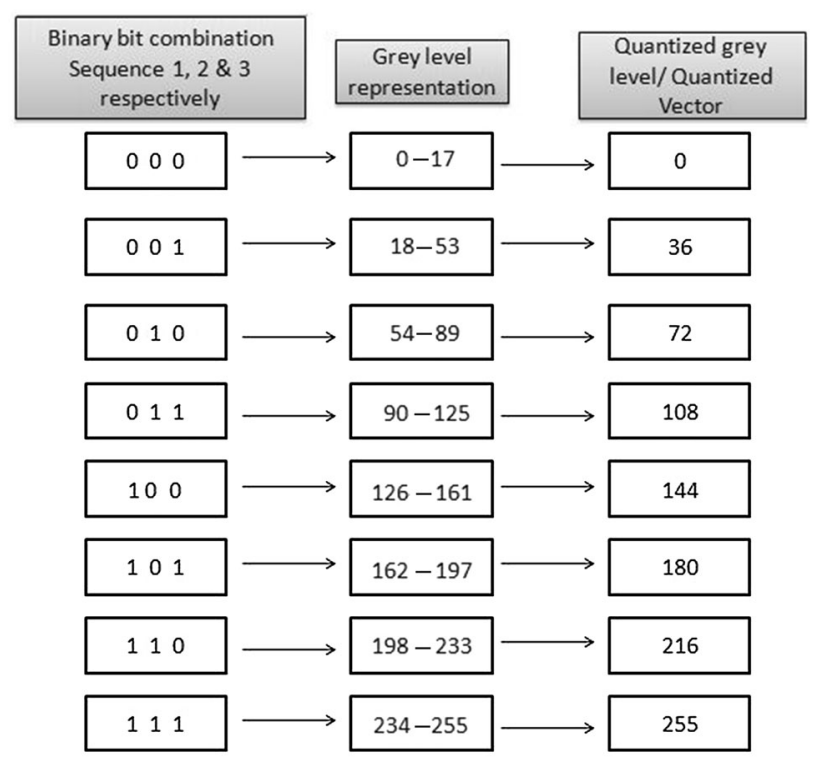

Figure 4. Encoding decision logic.

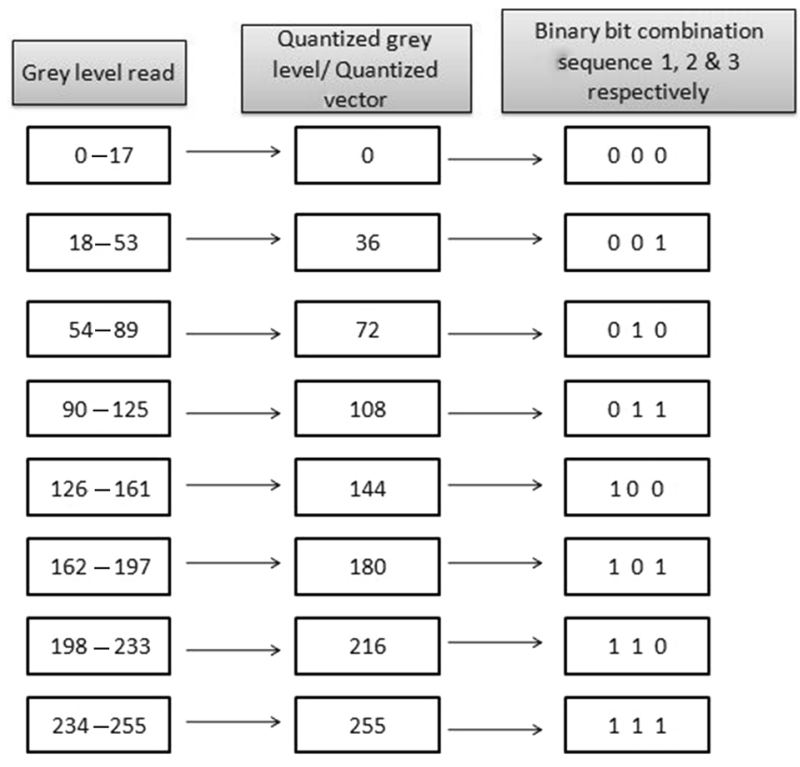

Figure 5. Decoding decision logic.

\subsection{Data decoding decision logic}

Decoding is the process by which data are recovered from encoded bits, that is, the barcode. The decoding is based on the concept of vector quantization where a particular range of intensity values corresponds to a single binary bit combination. The idea behind this is tolerance of intensity variation for a single binary bit combination. The intensity variation that occurred during printing and scanning is acceptable with a certain amount of tolerance. Figure 5 shows the decoding logic used for recovery of three independent data which can be extended to recover $n$ independent data.

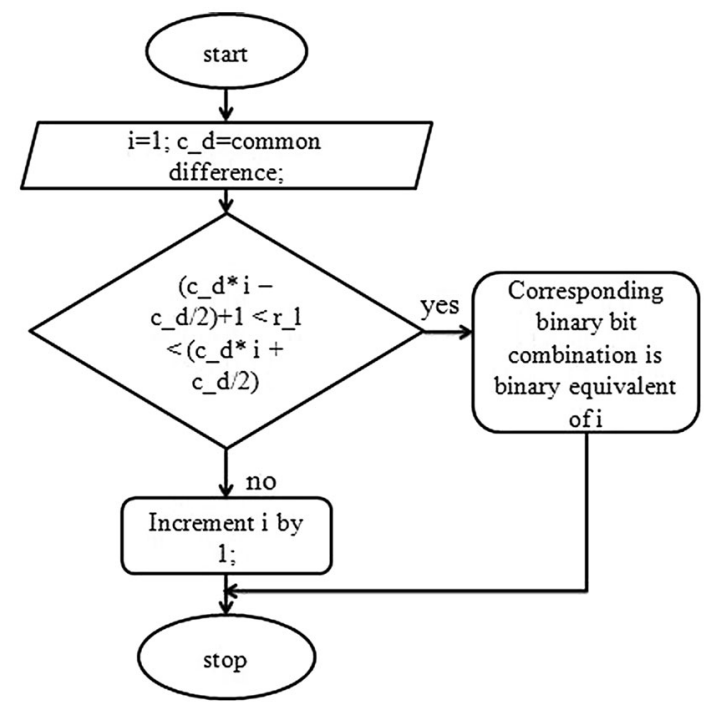

Figure 6. Decision logic for decoding ('c_d' is the common difference for grey level representation, ' $r$ ll' is grey level assigned and ' $\mathrm{i}$ ' is integer determining loop count). 
This proved that the proposed method provides acceptance to the intensity variations, as decoding uses a range of grey levels for decision rather than using a single grey level. Grey level intensity values are read from the scanned barcode and decision is made for extracting the independent data. Intensity variation may also change the intensity values in a particular module, in this case the average value of all the intensities in that module is taken, to make the further decision. Figure 6 shows the proposed decision making logic for extracting binary bit combination for respective monochrome barcodes while decoding a grey scale barcode.

\section{Implementation}

The barcode formulation with grey scale is a challenging task, specially for desktop and mobile applications. In this paper, encoding logic in figure 4 is used for creating grey scale barcode, that is, for combining $n$ independent barcodes into a single grey scale barcode. Moreover, 2D grey scale barcode is flexible according to the data capacity of the file. The data capacity of the $2 \mathrm{D}$ grey scale barcode is maximized using grey levels.

The key process in encoding is binarization of the data file and grouping of the bits. 2D grey scale barcodes have higher data capacity than black and white $2 \mathrm{D}$ barcodes. The data file may contain any type of characters, symbols or numerals. The block diagram representation of data encoding is shown in figure 3. Figure 7 shows the steps involved to generate 2D grey scale barcode. Three independent data inputs 'HELLO_WORLD!', 'welcome!!' and 'all_the_best' is considered to generate grey scale QR code. Figure 9 shows the generated grey scale barcode using the proposed decision logic.

The combination of different modules from different monochrome barcodes (bit combination) is replaced by a grey level. All the possible combination of modules (bits) for three sequences and their replacement using a grey level module is shown in figure 8 . Any possible combination of modules can be replaced by a value in a particular range, and multiple 2D Black and White barcode can be

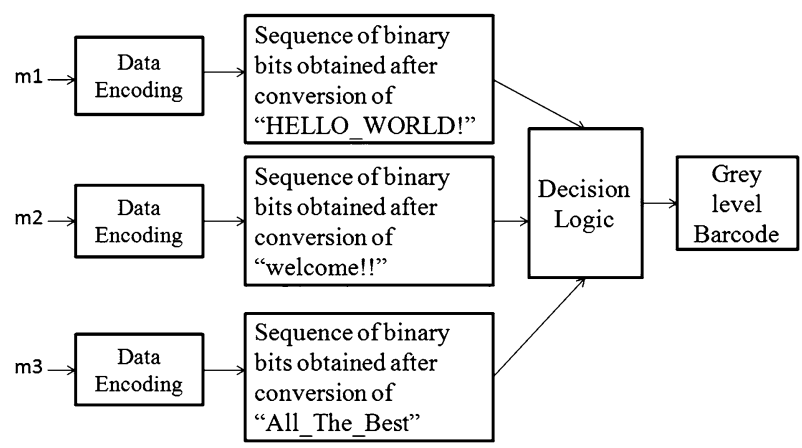

Figure 7. Proposed flow diagram to generate grey scale barcode.

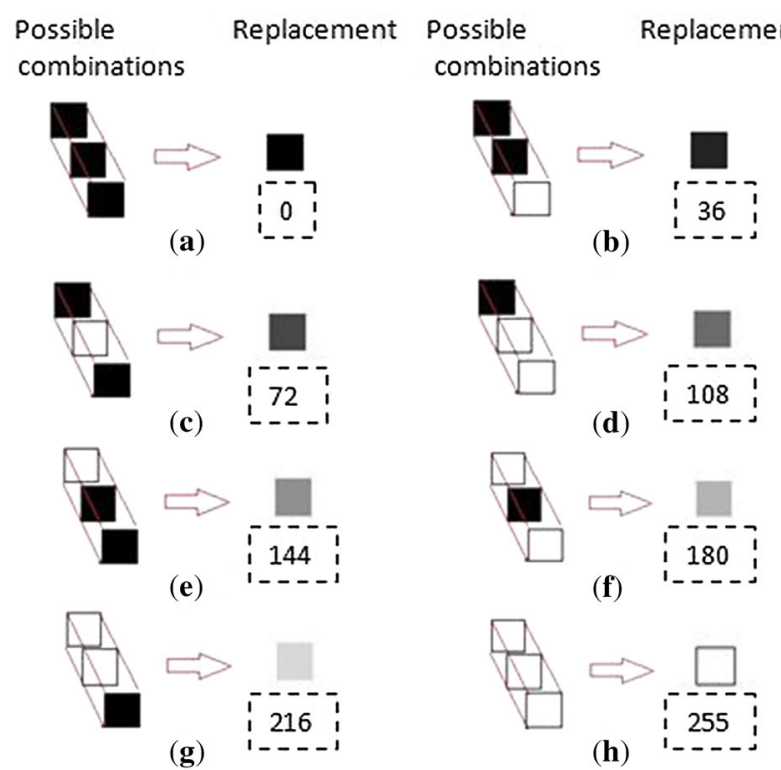

Figure 8. Replacing combination of binary modules by a grey scale module.

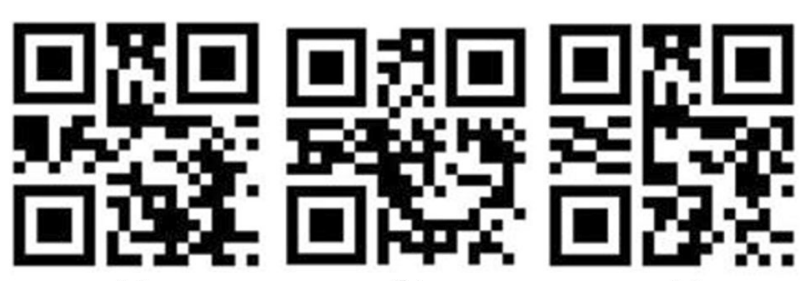

(a) (b)

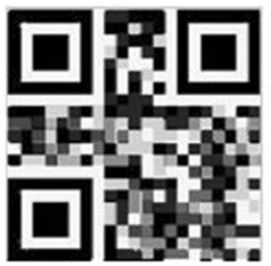

(d)
Figure 9. Example of proposed barcode method. Each barcode encodes the Data in QR format (a), (b) and (c) are monochrome QR barcode. (a) encodes HELLO_WORLD! (b) encodes welcome!! (c) encodes all_the_best and (d) represents the combined grey level barcode of the three barcodes (a), (b) and (c).

represented using a single grey scale barcode as shown in figure 9.

\subsection{Printing and scanning}

The basic reason behind proposing the idea of grey scale code over colour barcode is to overcome the problem of intensity variations in printing and scanning. The original grey scale barcode is first printed and then scanned and reliability of proposed barcode is tested. The scanned image is quite distorted, rough and pixel to pixel intensity 


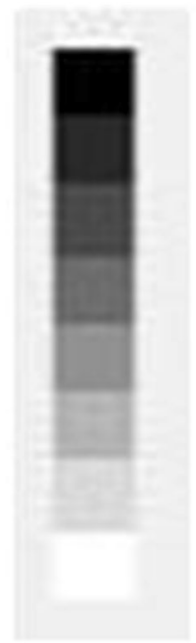

(a)

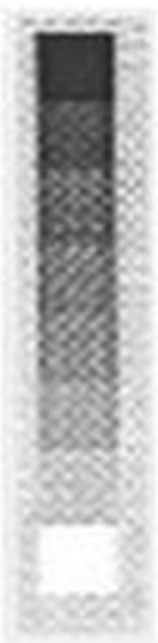

(b)
Figure 10. (a) shows the original (generated) image (b) is obtained by printing and then scanning the printed image.

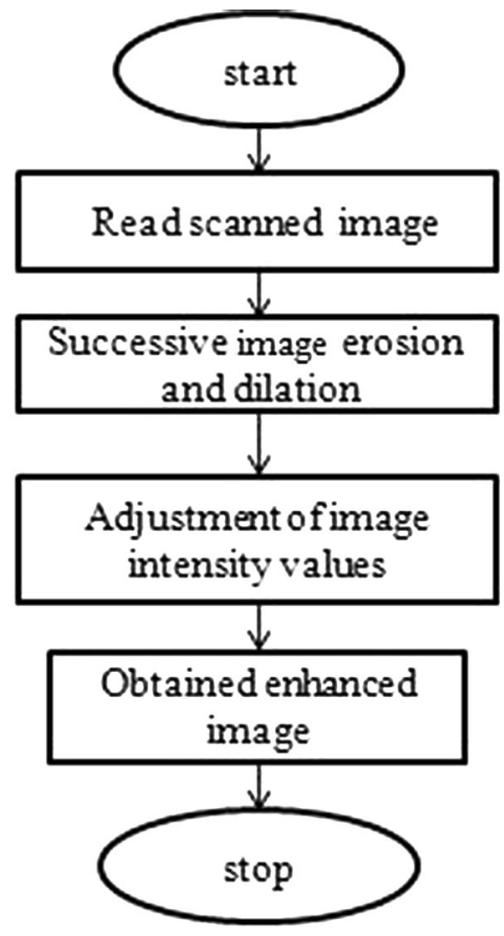

Figure 11. Algorithm to enhance scanned image.

variation from original intensity values as shown in figure 10. Figure 10a shows a grey scale image with 8 grey levels with corresponding quantized grey levels selected as given in figure 4 . The upper most module is intensity 0 , second module is intensity 36 , third module is intensity 72 and so on.

Our aim is to recover the original encoded data using this scanned image. First, the scanned image is smoothed and enhanced using the algorithm shown in figure 11.

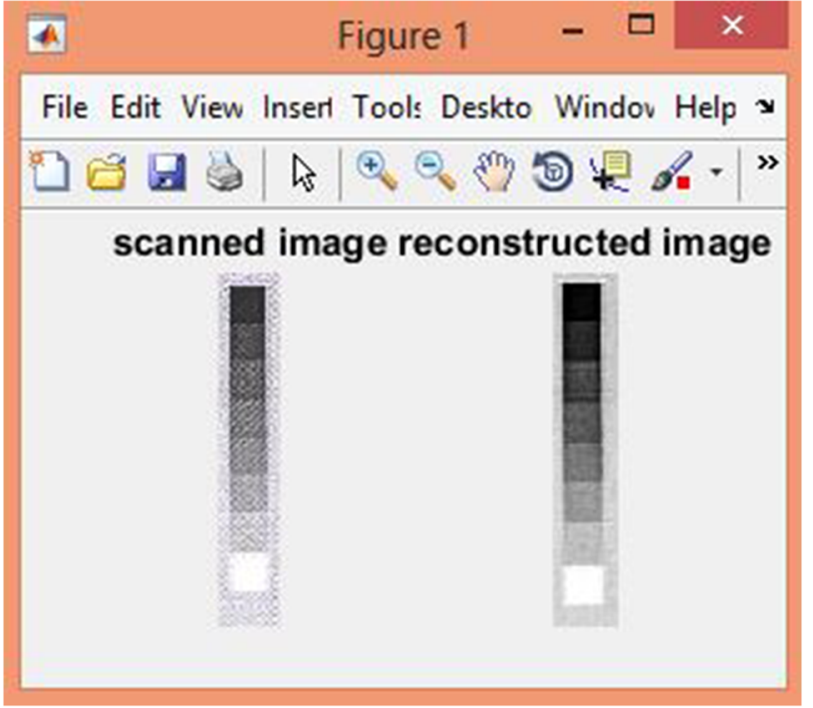

Figure 12. Enhanced scanned image.

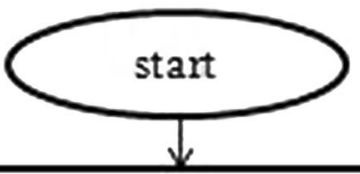

Find mean intensity value of each module

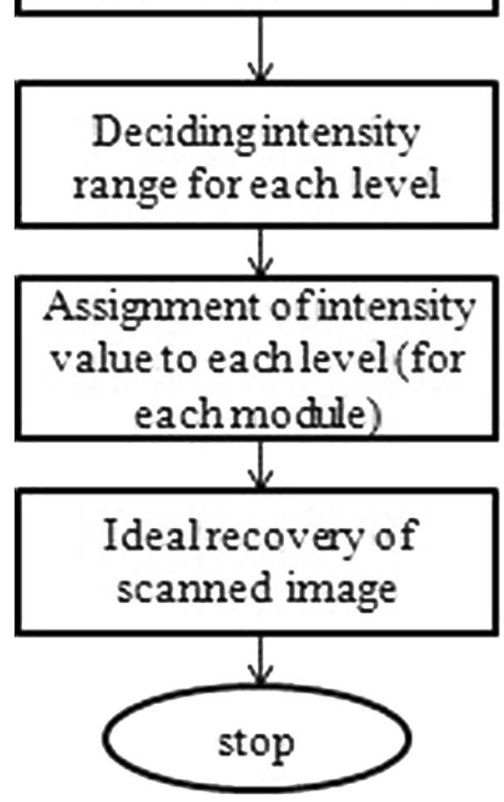

Figure 13. Ideal recovery of scanned image.

The algorithm proposed in figure 11 helps in enhancing the scanned image by using successive erosion and dilation and adjustment of intensity values. This algorithm is 
Table 1. Subjective analysis for intensity variation in various scanned images.

\begin{tabular}{|c|c|c|c|c|c|}
\hline $\begin{array}{l}\text { Intensity value range } \\
\text { (decision logic) }\end{array}$ & Levels & $\begin{array}{c}\text { Scanned image } 1 \\
\text { (intensity value observed) }\end{array}$ & $\begin{array}{c}\text { Scanned image } 2 \\
\text { (intensity value observed) }\end{array}$ & $\begin{array}{c}\text { Scanned image } 3 \\
\text { (intensity value observed) }\end{array}$ & $\begin{array}{c}\text { Intensity } \\
\text { value assigned }\end{array}$ \\
\hline $0-17$ & 0 & 7 & 8 & 6 & 0 \\
\hline $18-53$ & 1 & 38 & 38 & 37 & 36 \\
\hline 54-89 & 2 & 70 & 69 & 66 & 72 \\
\hline $90-125$ & 3 & 87 & 88 & 90 & 108 \\
\hline $126-161$ & 4 & 118 & 119 & 119 & 144 \\
\hline $162-197$ & 5 & 154 & 154 & 152 & 180 \\
\hline $198-233$ & 6 & 187 & 190 & 190 & 216 \\
\hline $234-255$ & 7 & 254 & 254 & 254 & 255 \\
\hline
\end{tabular}

applied to the scanned input image and an enhanced and better quality image is obtained as shown in figure 12 .

This enhanced image (reconstructed image) is then used to obtain original image using the algorithm in figure 13 . This algorithm is dependent on the variations observed in printing and scanning in various scanned images. The original intensity levels are assigned to a range of intensity depending on the possible variations that are observed in various scanned images.

Table 1 shows the intensity variation in different scanned images. Observation is made through various scanned images and original intensity levels are assigned to a range of intensity.

\section{Result and discussion}

\subsection{Generation of grey scale barcode}

The 2D grey scale barcode is developed employing vector quantization using 256 grey levels. The barcode developed using proposed method has higher data capacity as compared to monochrome QR code. Data capacity is increased upto $n$ times $(n \leq 8)$ as compared to black and white barcodes. As value of ' $n$ ' increases, the accuracy of decoding original data decreases. This is because the range of grey level representation decreases as indicated in figure 4 with increasing value of ' $n$ '. Each square module in the data set stores 3 bits here (can be generalized to ' $n$ ' bits).

The QR code has been implemented using MATLAB. The word HELLO_WORLD!, welcome!! and All_The_Best are given as the input.

First, the monochrome QR code for the input HELLO_WORLD!, "welcome!!", and "All_The_Best" is generated using the standard QR code generation method as shown in figure $14 \mathrm{a}, \mathrm{b}$ and $\mathrm{c}$ respectively. After this, the three monochrome barcodes are combined using grey level representation corresponding to the binary bit combination taking one bit from each barcode respectively. The bit taken from each barcode corresponds to the same module location in respective barcodes. This single grey scale barcode in figure $14 \mathrm{~d}$ embeds all the three monochrome barcodes in it.

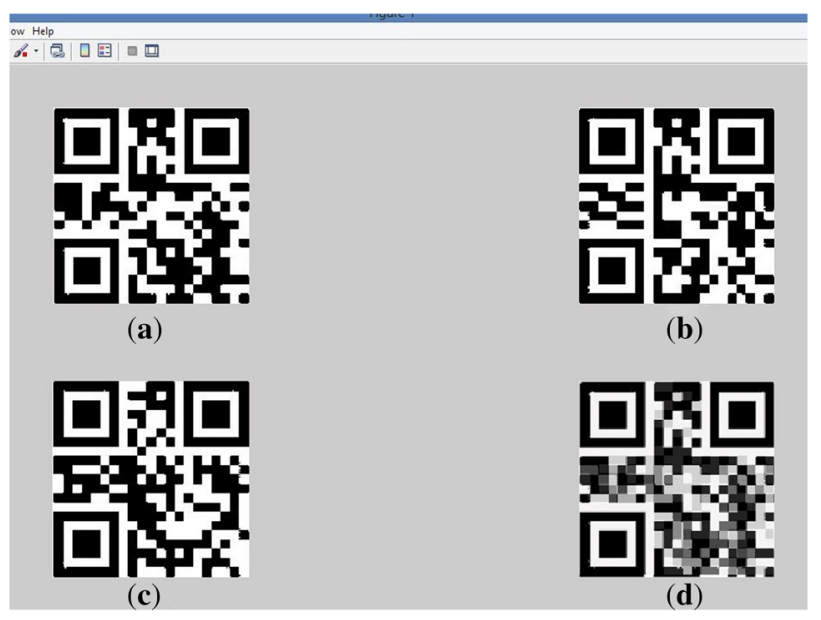

Figure 14. (a) Monochrome $\mathrm{QR}$ code for input data sequence 1 'HELLO_WORLD', (b) monochrome QR code for the input Data sequence 2 welcome!!, (c) monochrome QR code for the input data sequence 3 All_The_Best and (d) the grey scale QR code combining all the three independent data inputs in a single barcode.

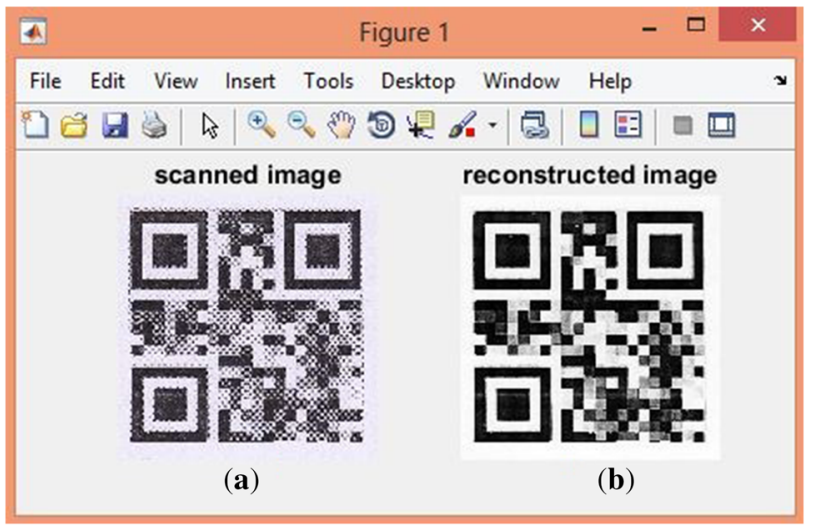

Figure 15. Reconstruction.

\subsection{Decoding results}

7.2a Printing and scanning: Figure 15a is the scanned image from printed barcode. This scanned image is 
reconstructed using the algorithm given in figure 11. The reconstructed image is smoother and has corrected intensity levels. The reconstructed image in figure $15 \mathrm{~b}$ shows enhanced image obtained from the scanned image.

The reconstructed image in figure $15 \mathrm{~b}$ is then used to get the original image using the algorithm described in figure 13. This image is identical to the original generated image, refer to the reconstructed original image in figure $16 \mathrm{~b}$. This image is used to perfectly decode the three sequences encoded in it.

Now, the grey scale QR code (reconstructed original image) is given as the input and three hidden and independent monochrome QR codes are obtained as shown in figure 17.

The output QR code 1 in figure 17 is decoded to obtain the corresponding data encoded in it, shown in figure 18 .

The output QR code 2 in figure 17 is decoded to obtain the corresponding data encoded in it, shown in figure 19 .

The output QR code 3 in figure 17 is decoded to obtain the corresponding data encoded in it, shown in figure 20 .

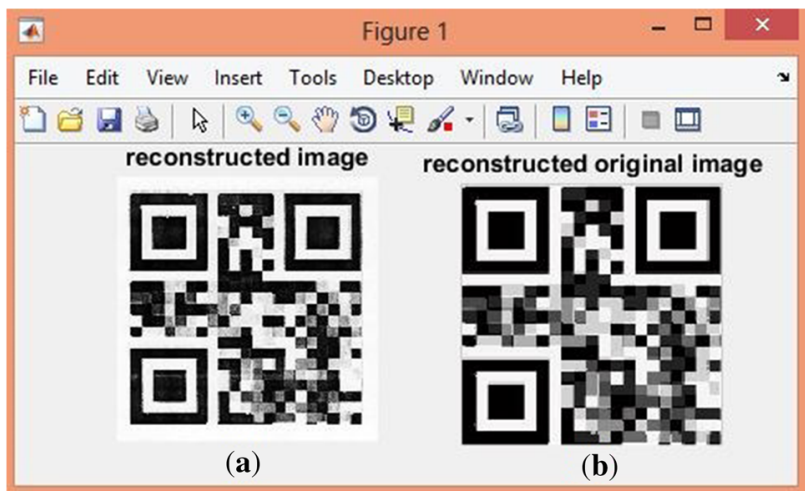

Figure 16. Reconstructed original image.

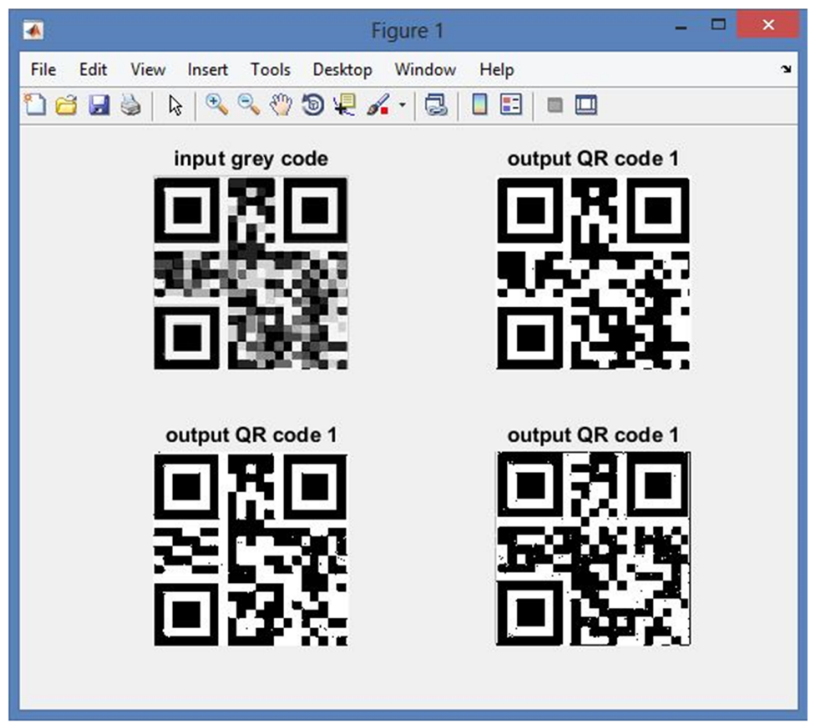

Figure 17. Decoding grey code to obtain three independent black and white QR code. 7.2b Decoding of distorted images: In order to simulate physical damage and dirt marks, some tests have been performed on 2D grey Barcode. The same text messages HELLO_WORLD!, All_The_Best, Welcome!! were contained in all codes. Various types of damaging were made on it [3]. Initially, the simulation of damage has been effected on the digital images of the barcodes. Figure 21 shows 2D grey scale QR code with different levels of damage (dirt or mark). Figure 21a shows a grey scale QR code without data loss, while figure $21 \mathrm{~b}, \mathrm{c}$ and $\mathrm{d}$ shows different examples of damages by means of overlapping extraneous information. Grey scale QR code of figure $21 \mathrm{~b}$ and $\mathrm{c}$ is readable. On the contrary, it is not possible to recover the encoded information from barcode related to image $21 \mathrm{~d}$, never in partial form.

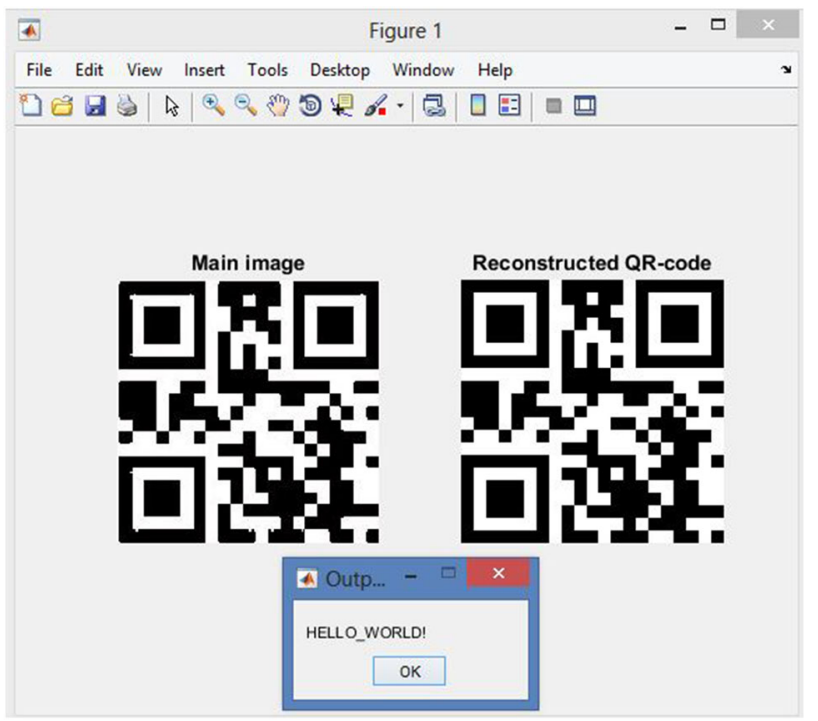

Figure 18. Decoded data sequence 1.

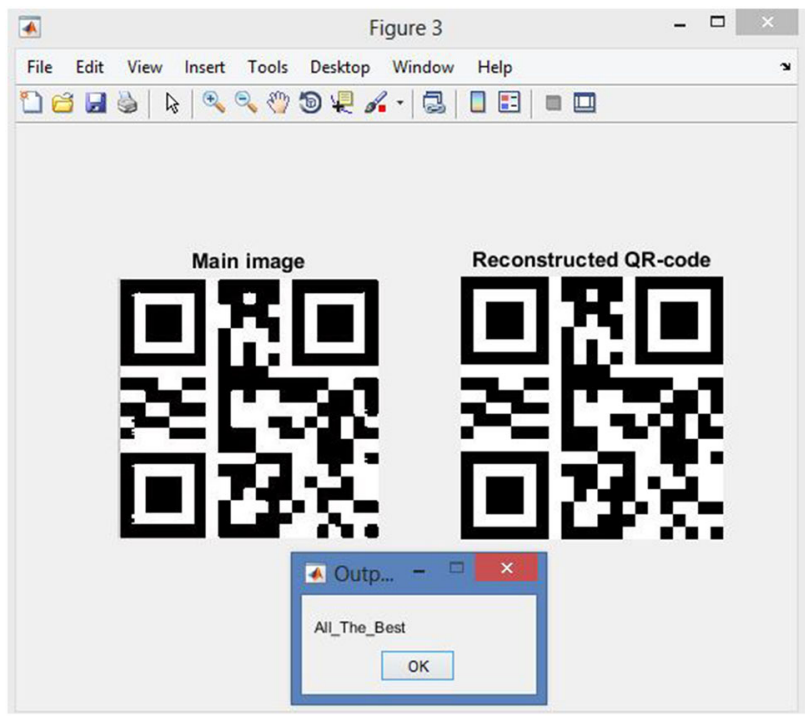

Figure 19. Decoded data sequence 2 . 


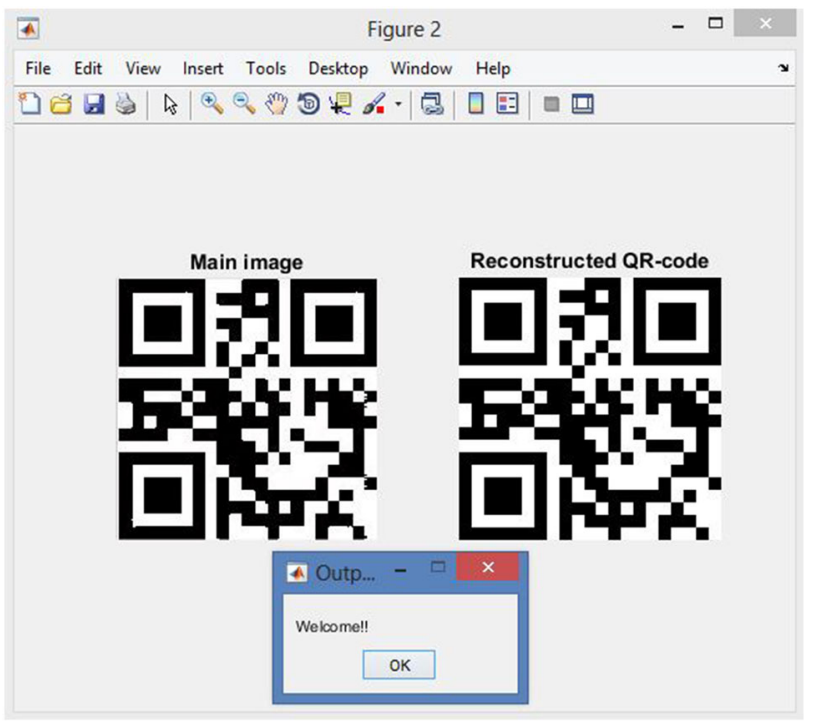

Figure 20. Decoded data sequence 3.

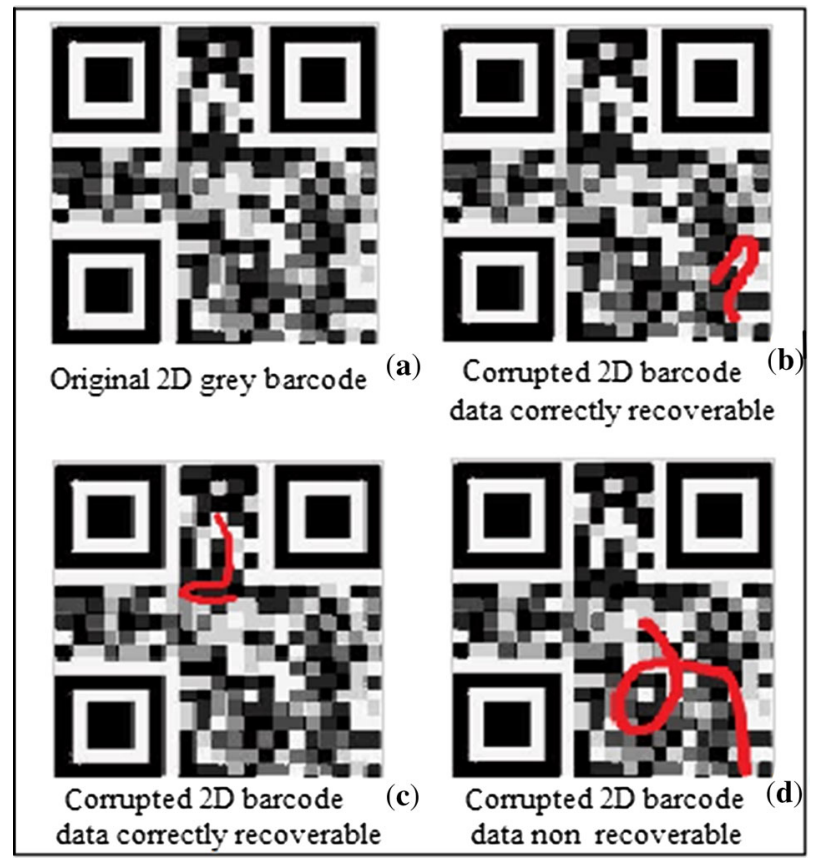

Figure 21. (a) Grey scale $\mathrm{QR}$ code without damage and (b) Readable grey scale $\mathrm{QR}$ code with data loss correction; (c and d) not readable grey scale $\mathrm{QR}$ code.

Figure 22 shows 2D grey code with different levels of damage, i.e., barcode area is damaged. Figure 22a shows a grey scale QR code without data loss, while figure $22 \mathrm{~b}$, c and $\mathrm{d}$ shows different examples of damages by means of various cuts. Grey scale QR code of figure $22 \mathrm{~b}$ is readable and figure $22 \mathrm{c}$ is partially recoverable. On the contrary, it is not possible to recover the encoded information from the barcode related to the image in figure $22 \mathrm{~d}$, never in partial form.

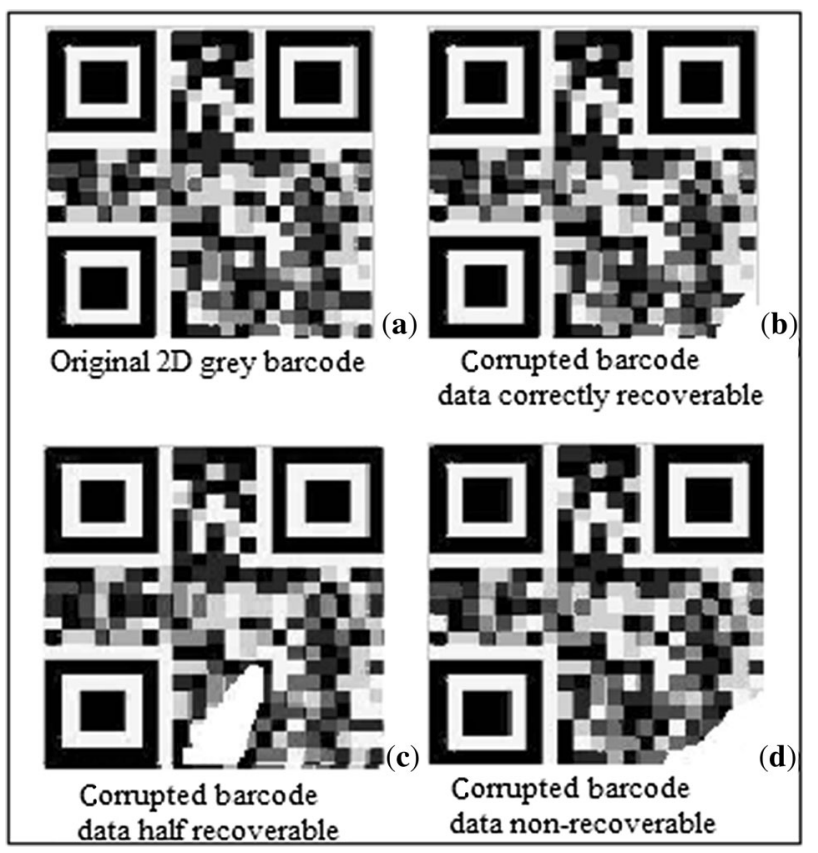

Figure 22. (a) Grey scale $\mathrm{QR}$ code without damage, (b) Readable grey scale QR code with data loss correction, (c) Grey scale QR code with partially recoverable and (d) not readable grey scale QR code.

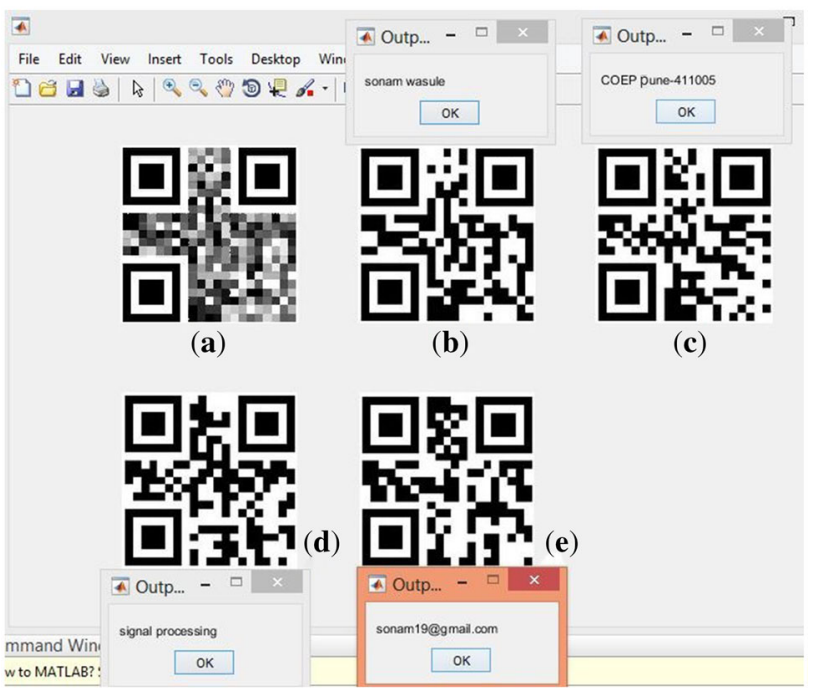

Figure 23. Increasing capacity of proposed barcode (a) Grey scale QR code embedding four data sequence, (b) Decoded data: Sonam Wasule, (c) Decoded data: COEP Pune-411005, (d) Decoded data: signal processing and (e) Decoded data: sonam191091@gmail.com.

\subsection{Increasing capacity of proposed barcode}

The grey scale QR code shown in figure 23a stores four independent data sequences in it. The grey scale QR code is then decoded to obtain all the four data sequences encoded in it. Thus, by this experiment we conclude that capacity of 
grey scale QR code is increased to 4 fold as compared with monochrome barcode. The colour QR code embeds three times more data than monochrome QR code, while grey scale QR code embeds four times more data as compared to monochrome QR code.

The grey scale $\mathrm{QR}$ code encoding three sequences forms eight possible combinations of three bits. Thus, each possible combination of bit is assigned a grey level as shown in figure 5 with acceptable tolerance during decoding. The tolerance decreases with increasing number of sequences. The data are accurately decoded for grey scale barcode embedding four data sequences. The grey scale QR code encoding five or more data sequence has a very less tolerance of grey levels and thus, less capable of recovering the data accurately after printing and scanning.

\section{Conclusion}

The grey scale $\mathrm{QR}$ code is processed to make the barcode independent of intensity and depth variation that occurs in colour barcode during printing and scanning. The proposed method increases the capacity of the grey scale QR code to ' $n$ ' fold but with varying accuracy. Experiments are performed on different grey scale barcodes and it is concluded that the barcode with 3 fold and 4 fold data capacity has higher accuracy. Though the capacity can be increased to 8 fold but we limit the capacity to 4 fold for accurate recovery. Accuracy of the barcode decreases with increasing value of $n(n \leq 8)$. We here conclude that, the proposed method solved the problem of intensity variation during printing and scanning and also increases the capacity of the barcode to $n(n \leq 4)$ fold. The proposed decoding algorithm is simpler as compared to algorithms used for decoding colour barcodes.

\section{References}

[1] Cadet Christopher M Boyls-White 2009 The mathematics of barcodes

[2] Ming Sun et al 2007 Research on optimization of encoding algorithm of Pdf417 brcode. China Agricultural University, Beijing, China, pp. 1261-1270

[3] Giuseppe Schirripa Spagnoloa et al 2012 New 2d barcode solution based on computer generated holograms: holographic barcode. In: Proceedings of the 5th International Symposium on Communications, Control and Signal Processing

[4] Ramya M and Jayasheela M 2014 Improved colour QR codes for real time applications with high embedding capacity. Int. J. Comput. Appl. (0975-8887) 91(8): 8-12

[5] Henryk Blasinski et al 2013 Per-colorant-channel colour barcodes for mobile applications: an interference cancellation framework. IEEE Trans. Image process. 22(4): 1498-1511

[6] Rinju Alice John and Kaamran Raahemifar 2015 Designing a 2D colour barcode. In: Proceeding of the IEEE 28th Canadian Conference on Electrical and Computer Engineering, pp. 297-301

[7] Orhan Bulan and Gaurav Sharma 2012 Improved colour barcodes via expectation maximization style interference cancellation. IEEE International Conference on Acoustics, Speech and Signal Processing, pp. 1509-1512

[8] Devi Parikh and Gavin Jancke 2000 Localization and segmentation of a $2 \mathrm{D}$ high capacity colour barcode. Microsoft Research Redmond, pp. 1-6 